\title{
Efeitos do exercício passivo precoce em cicloergômetro na espessura muscular do quadríceps femoral de pacientes críticos: estudo-piloto randomizado controlado
}

Effects of early passive cycling exercise on quadriceps femoris thickness in critically ill patients: a controlled randomized pilot study

Efectos del ejercicio pasivo precoz en cicloergómetro en el espesor muscular del cuádriceps femoral de pacientes criticos: un estudio piloto aleatorizado controlado

Maurício Tatsch Ximenes Carvalho', Everton Ludke², Dannuey Machado Cardoso³, Dulciane Nunes Paiva4, Janice Cristina Soares ${ }^{5}$, Isabella Martins de Albuquerque ${ }^{6}$

RESUMO | O objetivo deste estudo foi avaliar os efeitos do exercício passivo precoce em cicloergômetro na espessura muscular (EM) do quadríceps femoral (EMQ) de pacientes críticos admitidos em uma Unidade de Terapia Intensiva (UTI) de um hospital universitário terciário. O método utilizado foi um estudo-piloto randomizado controlado conduzido em uma amostra de 24 pacientes

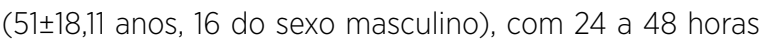
de ventilação mecânica (VM), aleatoriamente divididos em dois grupos: grupo-controle $(n=12)$, que recebeu a fisioterapia convencional; e grupo-intervenção $(n=12)$, que recebeu o exercício passivo em cicloergômetro, uma vez ao dia, durante o período de sete dias do protocolo, em adição à fisioterapia convencional. A EMQ foi mensurada através da ultrassonografia. A primeira medida ultrassonográfica foi realizada entre as primeiras 48 horas de VM e a segunda ao término do protocolo. Não houve diferenças significativas na EMQ esquerda $(27,29 \pm 5,86 \mathrm{~mm}$ vs. $25,95 \pm 10,89 \mathrm{~mm} ; \mathrm{p}=0,558)$ e direita $(24,96 \pm 5,59 \mathrm{~mm}$ vs $25,9 \pm 9,21 \mathrm{~mm} ; \mathrm{p}=0,682)$ do grupo-controle e na $E M Q$ esquerda $(27,2 \pm 7,38 \mathrm{~mm}$ vs $29,57 \pm 7,89 \mathrm{~mm} ; p=0,299)$ e direita $(26,67 \pm 8,16 \mathrm{~mm}$ vs $28,65 \pm 8,04 \mathrm{~mm} ; \mathrm{p}=0,381)$ do grupo-intervenção. Na comparação entre os grupos, não houve alterações significativas em relação à $E M Q$ esquerda (3,61 $\pm 1,07 \mathrm{~mm} ; \quad p=0,248)$ e a $E M Q$ direita $(2,75 \pm 0,85 \mathrm{~mm} ; \mathrm{p}=0,738)$. Os resultados deste estudopiloto demonstraram que a aplicação precoce do exercício passivo em cicloergômetro não promoveu mudanças significativas na espessura da camada muscular avaliada. No entanto, nossos achados sinalizam que a fisioterapia convencional foi capaz de preservar a EMQ de pacientes críticos admitidos em UTI.

Descritores | Modalidades de Fisioterapia; Unidades de Terapia Intensiva; Ultrassonografia; Terapia por Exercício; Deambulação Precoce.

\begin{abstract}
The objective of this study was to evaluate the effects of early passive cycling exercise on quadriceps femoris thickness (QFT) in critically ill patients admitted in the intensive care unit (ICU) of a tertiary care university hospital. A controlled randomized pilot study was conducted with a sample of 24 patients (51 \pm 18.11 years, 16 male), on mechanical ventilation (MV) from 24 to 48 hours, who were randomly divided into two groups: control group $(n=12)$,
\end{abstract}

Trabalho realizado na Unidade de Terapia Intensiva Adulto do Hospital Universitário de Santa Maria (HUSM) da Universidade Federal de Santa Maria (UFSM) - Santa Maria (RS), Brasil.

'Centro Universitário da Região da Campanha (URCAMP) - Bagé (RS), Brasil. E-mail: mauriciocarvalho@urcamp.edu.br.

Orcid: 0000-0001-6679-2854

2Universidade Federal de Santa Maria (UFSM) - Santa Maria (RS), Brasil. E-mail: evertonludke@gmail.com. Orcid: 0000-0002-3863-6069 3Universidade Federal do Rio Grande do Sul (UFRGS) - Porto Alegre (RS), Brasil. E-mail: dannuey@yahoo.com.br. Orcid: 0000-0003-2891-1028 ${ }^{4}$ Universidade de Santa Cruz do Sul (UNISC) - Santa Cruz do Sul (RS), Brasil. E-mail: dulciane@unisc.br. Orcid: 0000-0001-5629-3285 ${ }^{5}$ Hospital Universitário de Santa Maria - Santa Maria (RS), Brasil. E-mail: janicesoares9@yahoo.com.br. Orcid: 0000-0001-6991-2591 6Universidade Federal de Santa Maria (UFSM) - Santa Maria (RS), Brasil. E-mail: albuisa@gmail.com. Orcid: 0000-0002-7256-1881 
receiving conventional physical therapy; and an intervention one $(n=12)$, receiving passive cycle ergometer, once a day, throughout seven days of protocol, in addition to conventional physical therapy. The QFT was measured by ultrasonography. The first ultrasonographic measurement was performed within 48 hours after the start of MV, and the second at the end of the protocol. There were no significant differences in QFT of the left $(27,29 \pm 5,86 \mathrm{~mm}$ vs $25,95 \pm 10,89 \mathrm{~mm} ; \mathrm{p}=0,558)$ and right $(24,96 \pm 5,59 \mathrm{~mm}$ vs $25,9 \pm 9,21 \mathrm{~mm} ; \mathrm{p}=0,682)$ in the control group, and in QFT of the left $(27,2 \pm 7,38 \mathrm{~mm}$ vs $29,57 \pm 7,89 \mathrm{~mm} ; p=0,299)$ and right $(26,67 \pm 8,16 \mathrm{~mm}$ vs $28,65 \pm 8,04 \mathrm{~mm} ; \mathrm{p}=0,381)$ in the intervention group. There were no significant between-groups differences for left QFT $(3,61 \pm 1,07 \mathrm{~mm} ; \mathrm{p}=0,248)$ and right QFT $(2,75 \pm 0,85 \mathrm{~mm} ; \mathrm{p}=0,738)$. The results of this pilot study demonstrate that application of early passive cycle ergometer exercises has not significantly change the muscle layer thickness. However, our findings indicate that conventional physical therapy is able to preserve the quadriceps femoris thickness in critically ill patients admitted in ICU.

Keywords | Physical Therapy Modalities; Intensive Care Units; Ultrasonography; Exercise Therapy; Early Ambulation.

RESUMEN I El presente estudio tuvo como objetivo evaluar los efectos del ejercicio pasivo precoz en cicloergómetro en el espesor muscular (EM) del cuádriceps femoral (EMC) de pacientes críticos ingresados en una Unidad de Cuidados Intensivos (UCl) de un hospital universitario terciario. Se utilizó como método un estudio piloto aleatorizado controlado con una muestra de 24 pacientes (51ะ18,11 años, 16 varones), con 24 a 48 horas de ventilación mecánica (VM), quienes fueron divididos aleatoriamente en dos grupos: grupo de control ( $n=12)$, que recibió fisioterapia convencional; y grupo intervención ( $n=12$ ), que recibió el ejercicio pasivo en cicloergómetro una vez al día durante el período de protocolo de siete días, además de la fisioterapia convencional. El EMC se midió por ecografía. La primera medición ecográfica se realizó entre las primeras 48 horas de VM, y la segunda al final del protocolo. No hubo diferencias significativas en el EMC izquierdo (27,29 $\pm 5,86$ mm vs. 25,95 $\pm 10,89 m m$; $p=0,558)$ y derecho $(24,96 \pm 5,59 \mathrm{~mm}$ vs $25,9 \pm 9,21 \mathrm{~mm} ; \mathrm{p}=0,682)$ del grupo de control; y en el EMC izquierdo (27,2 $\pm 7,38 \mathrm{~mm}$ vs 29,57 $\pm 7,89 \mathrm{~mm}$; $p=0,299)$ y derecho $(26,67 \pm 8,16 \mathrm{~mm}$ vs $28,65 \pm 8,04 \mathrm{~mm} ; \mathrm{p}=0,381)$ del grupo intervención. En la comparación entre grupos, no hubo cambios significativos en el EMC izquierdo (3,61 $\pm 1,07 \mathrm{~mm} ; \mathrm{p}=0,248)$ y en el EMC derecho $(2,75 \pm 0,85 \mathrm{~mm}, \mathrm{p}=0,738)$. Los resultados de este estudio piloto demostraron que la aplicación precoz del ejercicio pasivo en cicloergómetro no promovió cambios significativos en el espesor de la capa muscular evaluada. Sin embargo, nuestros hallazgos indican que la fisioterapia convencional pudo preservar el EMC de pacientes críticos ingresados en la UCl.

Palabras clave | Modalidades de Fisioterapia; Unidades de Cuidados Intensivos; Ultrasonografía; Terapia por Ejercicio; Ambulación Precoz.

\section{INTRODUÇÃO}

O declínio rápido da massa muscular esquelética é o fator principal para deficiência física comum entre os pacientes críticos em ventilação mecânica $(\mathrm{VM})^{1}$. A disfunção muscular precoce ocorre em um período de horas a dias, especificamente nos primeiros 7 a 10 dias de internação na unidade de terapia intensiva (UTI), há um declínio na espessura muscular do quadríceps femoral (EMQ) ${ }^{2,3}$.

A quantificação da EM periférica pode ser realizada através da ultrassonografia (US) muscular. Esse instrumento representa uma modalidade atrativa para aplicabilidade precoce no cenário do paciente crítico, uma vez que se constitui de uma ferramenta segura, não invasiva, preditora do volume muscular, podendo avaliar a efetividade de intervenções ${ }^{4-6}$.

Entre os recursos utilizados na profilaxia da atrofia muscular do quadríceps femoral, destaca-se o cicloergômetro de membros inferiores. Tal dispositivo é utilizado em modo passivo, principalmente nos primeiros dias de internação na
UTI, permitindo a realização em pacientes com redução do nível de consciência ${ }^{7}$. Estudos prévios demonstraram que a realização de mobilização passiva contínua cíclica, além de constituir-se em uma atividade segura e factível, auxilia na recuperação da força muscular periférica de pacientes críticos internados em $\mathrm{UTI}^{8,9}$.

Partindo do pressuposto que a primeira semana de internação na UTI é um período decisivo no que tange à degradação da EM periférica ${ }^{2}$, principalmente do quadríceps femoral, torna-se necessária a aplicabilidade de recursos que previnam a atrofia muscular. Desse modo, o objetivo deste estudo foi investigar os efeitos da aplicação precoce do exercício passivo com o cicloergômetro associado à fisioterapia convencional na EMQ de pacientes internados em UTI.

\section{METODOLOGIA}

Ensaio clínico piloto randomizado com cegamento de avaliadores de desfecho, realizado na UTI Adulto 
do Hospital Universitário de Santa Maria (HUSM) da Universidade Federal de Santa Maria (UFSM), em Santa Maria, Rio Grande do Sul, Brasil, entre julho e outubro de 2015. Todos os participantes ou seus familiares assinaram o Termo de Consentimento Livre e Esclarecido antes de serem incluídos no estudo, conforme determina a Resolução no 466/2012 do Conselho Nacional de Saúde.

Foram incluídos pacientes de ambos os sexos, com idade superior a 18 anos, entre 24 a 48 horas de VM após a admissão na UTI, nível de sedação profundo avaliado através da escala Richmond Agitation Sedation Scale $^{10}$ (RASS=-4) e hemodinamicamente estáveis. Foram excluídos pacientes que estavam recebendo cuidados paliativos, pacientes amputados ou com fratura de membros inferiores, com doenças neuromusculares ou neurológicas, com sequelas motoras, assim como pacientes incapazes de utilizar o cicloergômetro devido à pré-existência de disfunções articulares e/ou musculoesqueléticas.

\section{Randomização e intervenção}

Os pacientes recrutados para o estudo foram avaliados por meio de registros clínicos, informações demográficas, razão primária de admissão na UTI e do escore acute physiology and chronic health evaluation II (Apache II) ${ }^{11}$.

Os indivíduos foram alocados a partir de tabela de números aleatórios, gerada por computador com sequência de randomização criada pelo software Random Number Generator (Pro v2.00, Segobit, Issaquah, WA, EUA). Todos os participantes receberam a intervenção por dois fisioterapeutas. Devido à natureza da intervenção, os fisioterapeutas responsáveis pela realização da mobilização precoce em cicloergômetro não foram cegados em relação à randomização dos pacientes. No entanto, os avaliadores de desfecho foram mantidos cegos quanto à alocação, tendo em vista que não participaram das intervenções do estudo.

Os pacientes que preencheram os critérios de inclusão foram alocados para o grupo-intervenção (GI) ou grupocontrole (GC). O GC foi submetido à fisioterapia convencional, enquanto o GI recebeu adicionalmente sessões de exercício passivo através da utilização do cicloergômetro de membros inferiores (MOTOmed letto 2, RECK-Technik GmbH \& Co.KG, Betzenweiler, Alemanha). Desse modo, foram realizadas sessões com o cicloergômetro no modo passivo, com o paciente em decúbito dorsal e elevação da cabeceira a $30^{\circ}$, por 20 minutos, cadência fixa de 20 ciclos/min, uma vez ao dia, durante a primeira semana de internação na UTI. Com o propósito de garantir a realização do exercício passivo, a tela do equipamento, a qual possibilita a visualização/ análise do treino e detecta movimentos ativos, foi constantemente monitorada durante o protocolo.

A fisioterapia convencional (respiratória e motora) foi realizada por fisioterapeutas da UTI, duas vezes ao dia, por aproximadamente 30 minutos, no período de 7 dias. O protocolo incluiu manobras de vibrocompressão, hiperinsuflação pelo ventilador mecânico e aspiração traqueal, quando necessária, além de exercícios motores de membros superiores e inferiores, passivos e ativoassistidos, conforme a evolução clínica do paciente.

Durante e após a realização do protocolo, foram monitorados constantemente os parâmetros cardiovasculares, tais como: saturação periférica de oxigênio $\left(\mathrm{SpO}_{2}\right)$, frequência cardíaca $(\mathrm{FC})$, pressão arterial média (PAM), pressão arterial sistólica e pressão arterial diastólica, de forma não invasiva, pela observação do monitor multiparamétrico DX 2022 (Dixtal Biomédica, Manaus, Brasil). Foram utilizados como critérios de interrupção do protocolo os seguintes parâmetros: instabilidade hemodinâmica $(\mathrm{PAM}<60$ ou $>125 \mathrm{mmHg}$ ), $\mathrm{SPO}_{2}<88 \%, \mathrm{FC}>130 \mathrm{bpm}$ ou $<40 \mathrm{bpm} \mathrm{e}$ sinais de desconforto respiratório.

\section{Avaliação da espessura muscular do quadríceps femoral}

A EMQ foi avaliada através da US de alta resolução (Ultrassom Mindray, DP-2200 portátil), em modo $\mathrm{B}$, com transdutor ecocardiológico microconvexo (65C15EA 5,0-9,0MHz, 4W). A avaliação inicial da EMQ foi realizada entre as primeiras 48 horas de VM e a segunda após os 7 dias de mobilização (término do protocolo).

O protocolo de avaliação através da US foi baseado no estudo de Fivez et al. ${ }^{12}$. O paciente foi posicionado em decúbito dorsal com os membros inferiores estendidos. Sequencialmente, o transdutor foi revestido com um gel ultrassônico e posicionado perpendicularmente ao ponto médio do quadríceps femoral identificado através de uma fita métrica. Após a detecção da imagem ultrassonográfica em resolução adequada para visualização muscular (Figura 1), ela foi capturada, e as mensurações, expressas em milímetros, foram realizadas. A EMQ foi determinada através de imagens transversais, medindo a distância da borda externa do fêmur até a aponeurose superior do músculo reto femoral. 


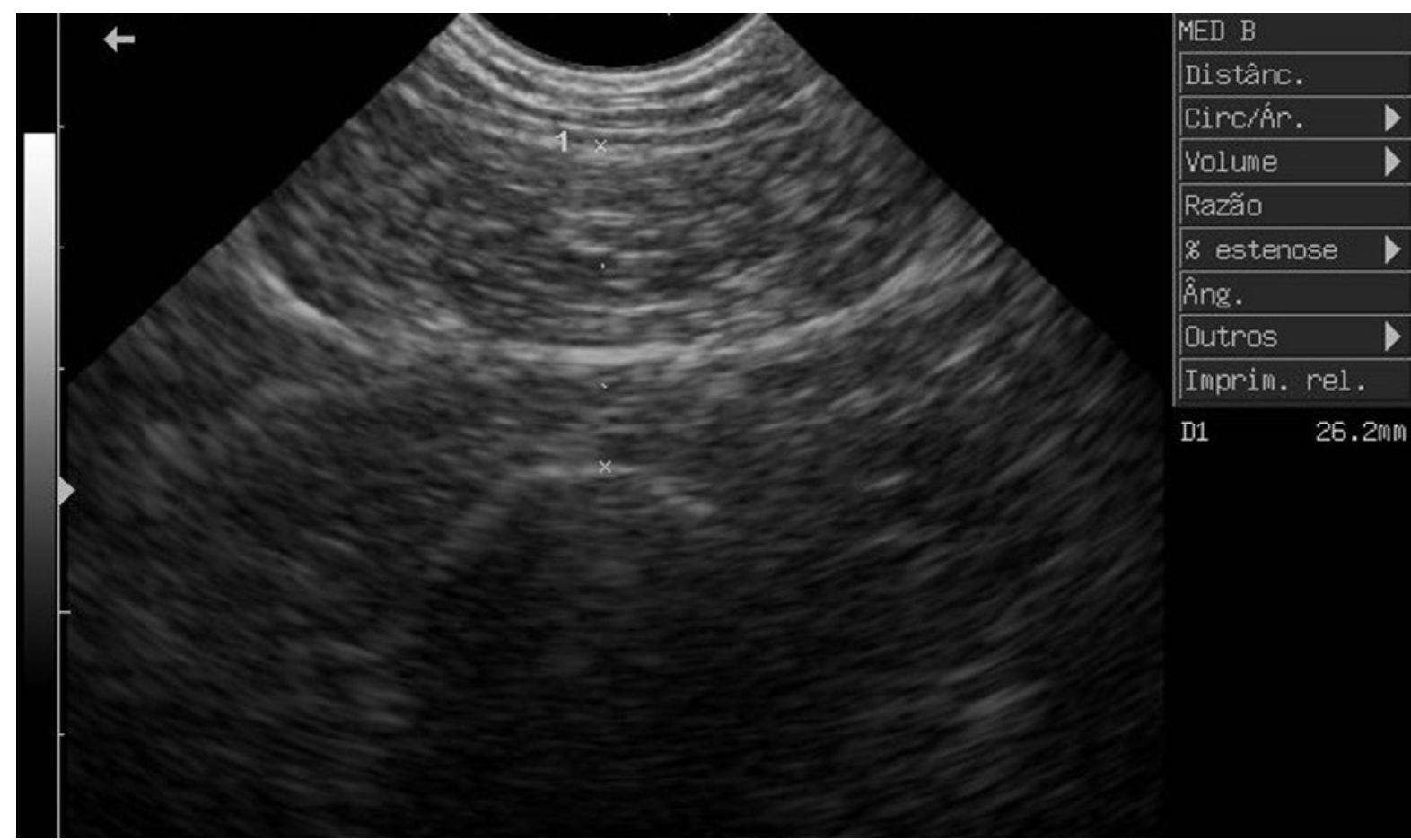

Figura 1. Espessura muscular do quadríceps femoral avaliada através da ultrassonografia em paciente do grupo-intervenção

\section{Cálculo do tamanho amostral}

A amostra deste estudo-piloto foi utilizada para inferência amostral do ensaio clínico randomizado. Estimado para obtenção de um nível de significância de $5 \%(\mathrm{p}<0,05)$ e poder de $80 \%$ (programa WinPepi, versão 10.5), considerando uma diferença de $2,37 \mathrm{~mm}$ na EMQ e um número amostral de 64 pacientes em cada grupo.

\section{Análise estatística}

A análise estatística foi realizada utilizando o programa IBM SPSS Statistics, versão 20.0 (IBM Corporation, Armonk, NY, EUA). A normalidade das variáveis foi avaliada pelo teste de Shapiro-Wilk. As variáveis contínuas foram apresentadas na forma de média \pm desvio-padrão e intervalo de confiança de $95 \%$ (IC 95\%), enquanto as categóricas foram apresentadas em frequências absolutas e porcentagens. Para comparar os momentos pré e pós-intervenção intragrupo, foi utilizado o teste $\mathrm{t}$-Student pareado. A comparação entre grupos foi realizada utilizando Anova de duas vias seguidos pelo post-hoc de Bonferroni. O tamanho do efeito foi determinado a partir do $f$ de Cohen para comparar os grupos e classificá-los como grande, moderado e pequeno $^{13}$. Para efeito de significância estatística, foi considerado um valor de $\mathrm{p}<0,05$.

\section{RESULTADOS}

No período estudado, foram admitidos 76 pacientes na UTI Adulto da instituição, sendo que destes, 32 preencheram os critérios de inclusão, sendo randomizados em GC (n=16) e GI ( $n=16)$. Posteriormente, houve óbito de 4 pacientes do GC e 4 pacientes do GI. Dessa forma, a amostra final foi composta por 24 pacientes, totalizando 12 em cada grupo (Figura 2).

A Tabela 1 apresenta a caracterização geral da amostra, a qual foi homogênea na maioria das variáveis, exceto em relação à variável sexo. O GI apresentou um número maior de homens em comparação ao GC (p=0,009). Durante o estudo, não houve a necessidade de interromper o protocolo e nem foi observado qualquer evento adverso durante e após sua aplicação.

A EMQpermaneceu inalterada em ambos os grupos após a implementação do protocolo. Não houve diferenças significativas na EMQ esquerda $(27,29 \pm 5,86 \mathrm{~mm}$ vs. 25,95 $\pm 10,89 \mathrm{~mm} ; \mathrm{p}=0,558$; IC 95\%: $-3,53$ a 6,20) e direita $(24,96 \pm 5,59 \mathrm{~mm}$ vs. $25,9 \pm 9,21 \mathrm{~mm} ; \mathrm{p}=0,682 ; \mathrm{IC} 95 \%:-5,82$ a 3,95$)$ do GC; e na EMQ esquerda $(27,2 \pm 7,38 \mathrm{~mm}$ vs. 29,57 $\pm 7,89 \mathrm{~mm} ; \mathrm{p}=0,299$; IC 95\%: $-6,92$ a 2,34$)$ e direita $(26,67 \pm 8,16 \mathrm{~mm}$ vs. $28,65 \pm 8,04 \mathrm{~mm}$; $\mathrm{p}=0,381$; IC $95 \%$ : $-6,77$ a 2,80) do GI. Na comparação entre os grupos, não houve alterações significativas em relação à EMQ esquerda $(3,61 \pm 1,07 \mathrm{~mm} ; \mathrm{p}=0,248 ; \mathrm{IC} 95 \%:-4,16$ a 11,40$)$ e a EMQ direita $(2,75 \pm 0,85 \mathrm{~mm} ; \mathrm{p}=0,738$; IC 95\%: -4,69 a 10,21) com tamanho de efeito grande $(f$ de Cohen=0,634) (Figura 3 ). 


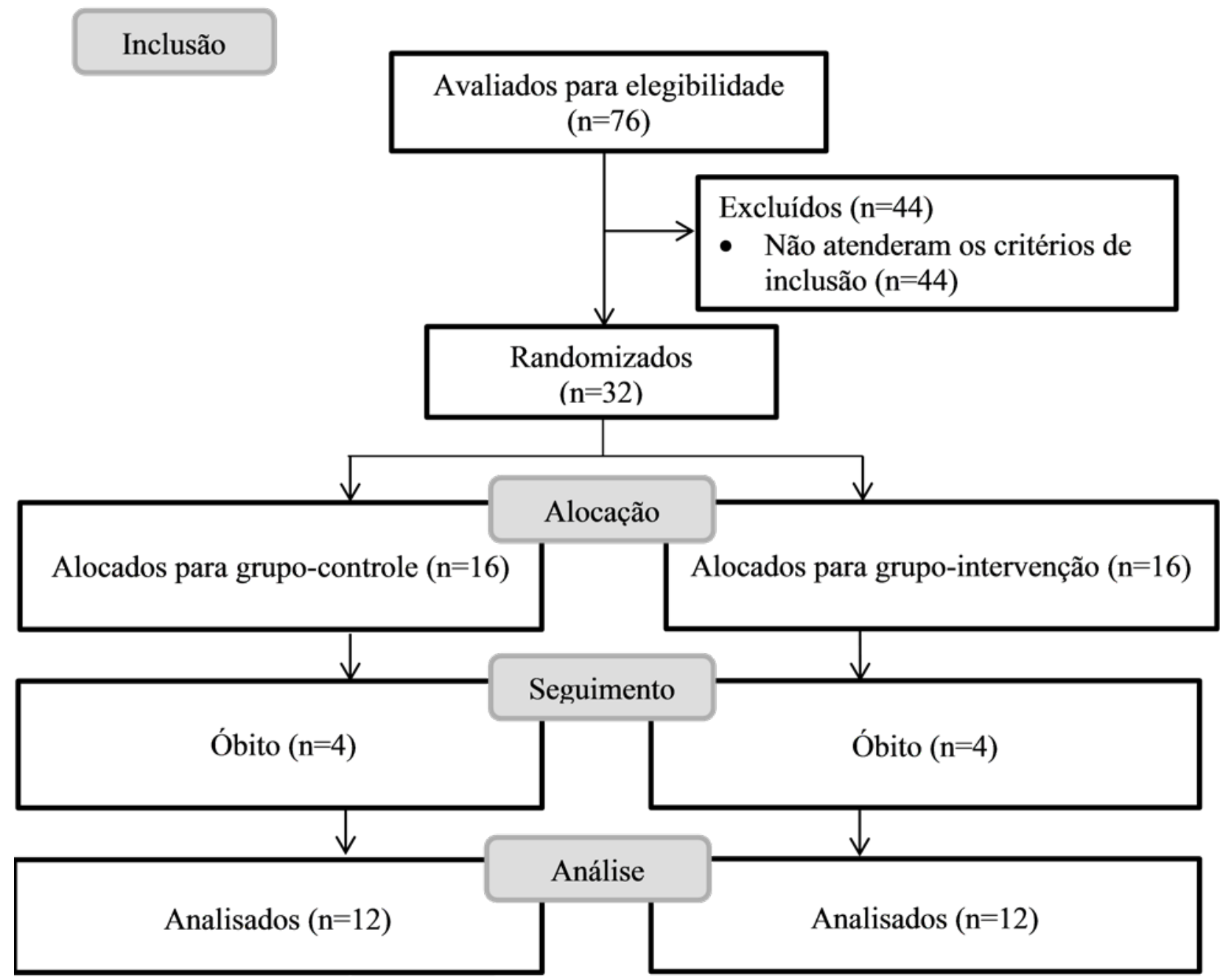

Figura 2. Fluxograma do estudo

Tabela 1. Características clínicas e demográficas dos pacientes

$\begin{array}{lcc}\text { Variáveis } & \text { Grupo-controle (n=12) } & \text { Grupo-intervenção (n=12) } \\ \text { Idade (anos) } & 54,17 \pm 16,71 & 47,83 \pm 19,61 \\ \text { Sexo masculino, }(n / \%)^{*} & 5(31,2) & 11(68,8) \\ \text { IMC }\left(\mathrm{kg} / \mathrm{m}^{2}\right) & 25,58 \pm 5,93 & 25,58 \pm 4,84 \\ \text { Escore APACHE II } & 16,00 \pm 5,84 & 14,42 \pm 6,25 \\ \text { Razão primária de admissão na UTI (n/\%)* } & & 1(8,33) \\ \text { Cardíaca } & 0(0,0) & 2(16,66) \\ \text { Abdominal } & 5(41,66) & 5(41,66) \\ \text { Neurológica } & 3(25) & 0(0,0) \\ \text { Respiratória } & 2(16,66) & 4(33,33) \\ \text { Outras } & 2(16,66) & 0(0) \\ \text { Medicamentos (n/\%)* } & & 3(25,0) \\ \text { Bloqueadores neuromusculares } & & 5(0) \\ \text { Corticosteróides } & 5(41,7) & 5(41,7) \\ \text { Vasopressores } & 5(41,7) & \end{array}$




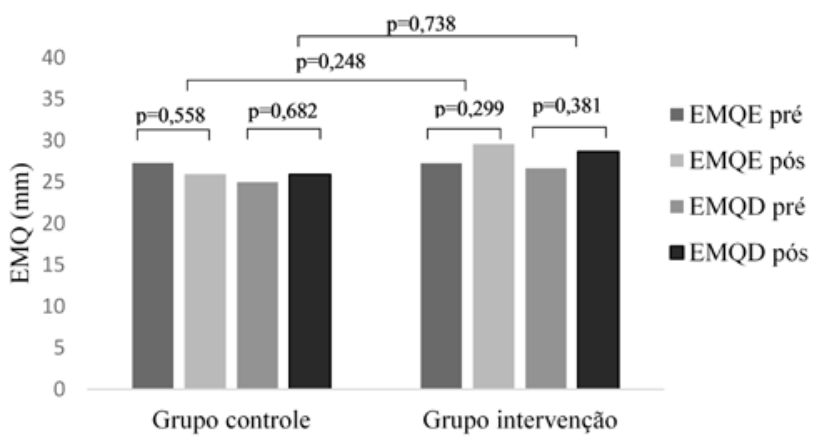

Figura 3. Espessura muscular do quadríceps femoral esquerdo e direito (EMQE e EMQD) avaliada

Medição através de ultrassonografia, antes e depois da implementação do protocolo do estudo. Teste t-Student intragrupo e Anova de duas vias seguido pelo pós-teste de Bonferroni entre os grupos.

\section{DISCUSSÃO}

Para o nosso conhecimento, este estudo-piloto randomizado é um dos primeiros a investigar os efeitos da aplicação precoce do exercício passivo, através do cicloergômetro, associado à fisioterapia convencional na EMQ de pacientes críticos. Após a implementação do protocolo, não houve alterações significativas na espessura da camada muscular tanto no GC quanto no GI, no entanto, infere-se que a fisioterapia convencional, realizada em ambos os grupos, promoveu a preservação da EMQ.

Estudos conduzidos em UTI têm demonstrado que $17 \%$ a $30 \%$ da massa muscular pode ser perdida nos primeiros 10 dias de internação do doente crítico $^{3,14}$. Tendo em vista a escassez de evidências a respeito dos efeitos do exercício passivo precoce em cicloergômetro na EMQ é importante mencionar o ensaio clínico piloto randomizado conduzido por Gruther et al..$^{15}$, no qual foi investigada, durante a primeira semana de internação na UTI, a eficácia da estimulação elétrica neuromuscular na prevenção da atrofia muscular periférica, no entanto, foi evidenciada uma diminuição significativa da EMQ, demonstrando que a intervenção precoce não preveniu a perda de massa muscular. No nosso estudo, a mobilização realizada através do cicloergômetro não promoveu efeitos adicionais ao protocolo de fisioterapia convencional, em contrapartida, inferese que a implementação desse protocolo preservou a EMQ. Uma possibilidade para explicar tal achado é que, neste estudo, todos os pacientes receberam fisioterapia precocemente, durante a primeira semana de permanência na UTI, especificamente iniciada nas primeiras 48 horas de internação. Além desse fator, infere-se que, provavelmente, ocorreu uma atenuação do estresse oxidativo ${ }^{16}$, a manutenção das propriedades intrínsecas de contratilidade do músculo ${ }^{17}$ e a ativação de citocinas anti-inflamatórias inibidoras dos mecanismos responsáveis pela deterioração muscular periférica ${ }^{18}$, no entanto, esses desfechos não foram avaliados neste estudo.

Estudos desenvolvidos em UTI têm demonstrado que a implementação da reabilitação precoce promove um estímulo anabólico nas fibras musculares periféricas ${ }^{19,20}$. No estudo conduzido por Burtin et al. ${ }^{21}$, foi observado um aumento da força do quadríceps após a combinação de mobilização passiva e ativa associada ao uso precoce do cicloergômetro. Neste estudo, todos os pacientes estavam em um nível de sedação profundo (escala de agitação e sedação de Richmond=-4), fator que impossibilitou a aferição da força muscular. Nesse contexto, conforme descrito por Puthucheary et al. ${ }^{22}$, a ultrassonografia pode substituir outros recursos avaliadores da força muscular periférica, como o Medical Research Council, especialmente em períodos nos quais os pacientes são incapazes de seguir comandos verbais, além desse aspecto, trata-se de uma ferramenta promissora utilizada para avaliação musculoesquelética e tal êxito se deve à minimização do viés gerado pelas convencionais medidas antropométricas ${ }^{23-26}$.

Em nossa amostra, houve um predomínio de homens, considerando este fator, indivíduos do sexo masculino, fisiologicamente, apresentam maior massa muscular em comparação as mulheres, no entanto, ressalta-se que esse aspecto não promove um efeito protetor em relação à atenuação da $\mathrm{EM}^{27}$. No estudo observacional conduzido por Turton et al. ${ }^{1}$, no qual similarmente houve maior proporção de homens, as avaliações ultrassonográficas realizadas, nos primeiros 10 dias de permanência na UTI, demonstraram um declínio significativo da EMQ.

Nosso estudo apresenta algumas limitações. Primeiramente, o nosso desfecho se restringiu à avaliação da EMQ na primeira semana de internação na UTI, portanto não podemos confirmar se o achado da preservação da EMQ se manteria até a alta hospitalar. Em segundo lugar, os demais parâmetros envolvidos na arquitetura muscular, tais como ângulo de penação, comprimento do fascículo e área de secção transversal, não foram aferidos, pois na literatura a maioria dos estudos 
realizados com pacientes críticos utilizam exclusivamente o parâmetro EMQ ${ }^{12,15,26,27}$. Em terceiro lugar, em uma parcela da amostra foram administrados corticosteroides, no entanto, não é bem estabelecida a relação casual entre disfunções neuromusculares e uso de corticosteroides, provavelmente em função dessas disfunções estarem relacionadas a mecanismos mais complexos, tais como: dose, tempo e concomitante controle glicêmico ${ }^{28}$.

\section{CONCLUSÃO}

Até o presente momento, os resultados deste estudopiloto demonstraram que a aplicação precoce do exercício passivo, através do cicloergômetro, associado à fisioterapia convencional, não promoveu alterações espessura muscular do quadríceps femoral. No entanto, nossos achados sinalizam que a fisioterapia convencional preservou a EM de pacientes críticos na primeira semana de permanência na UTI. Espera-se que o aumento do tamanho amostral indique resultados significativos quanto ao aumento da EM com uso do cicloergômetro passivo, visto que houve um tamanho do efeito grande com esta intervenção.

\section{REFERÊNCIAS}

1. Turton P, Hay R, Taylor J, McPhee J, Welters I. Human limb skeletal muscle wasting and architectural remodeling during five to ten days intubation and ventilation in critical care: an observational study using ultrasound. BMC Anesthesiol. 2016;16(1):119. doi: 10.1186/s12871-016-0269-z

2. Puthucheary ZA, Rawal J, McPhail M. Acute skeletal muscle wasting in critical illness. JAMA. 2013;310(15):1591-600. doi: 10.1001/jama.2013.278481

3. Files DC, Sanchez MA, Morris PE. A conceptual framework: the early and late phases of skeletal muscle dysfunction in the acute respiratory distress syndrome. Crit Care. 2015;19(1):266. doi: 10.1186/s13054-015-0979-5

4. Parry SM, El-Ansary D, Cartwright MS, Sarwal A, Berney S, Koopman R, et al. Ultrasonography in the intensive care setting can be used to detect changes in the quality and quantity of muscle and is related to muscle strength and function. J Crit Care. 2015;30(5):9-14. doi: 10.1016/j.jcrc.2015.05.024

5. Sarwal A, Parry SM, Berry MJ, Hsu FC, Lewis MT, Justus NW, et al. Interobserver reliability of quantitative muscle sonographic analysis in the critically ill population. J Ultrasound Med. 2015;34(7):1191-200. doi: 10.7863/ultra.34.7.1191

6. Hadda V, Khilnani GC, Kumar R, Dhunguna A, Mittal S, Khan MA, et al. Intra- and inter-observer reliability of quadriceps muscle thickness measured with bedside ultrasonography by critical care physicians. Indian J Crit Care Med. 2017;21(7):448-52. doi: 10.4103/ijccm.IJCCM_426_16
7. Santos LJ, Lemos FA, Bianchi T, Sachetti A, Dall'Acqua AM, Naue WS, et al. Early rehabilitation using a passive cycle ergometer on muscle morphology in mechanically ventilated critically ill patients in the Intensive Care Unit (MoVe-ICU study): study protocol for a randomized controlled trial. Trials. 2015;16:383. doi: 10.1186/s13063-015-0914-8

8. Kho ME, Martin RA, Toonstra AL, Zanni JM, Mantheiy EC, Nelliot A, et al. Feasibility and safety of in-bed cycling for physical rehabilitation in the intensive care unit. J Crit Care. 2015;30(6):1-5. doi: 10.1016/j.jcrc.2015.07.025

9. Machado AS, Pires-Neto RC, Carvalho MTX, Soares JC, Cardoso DM, Albuquerque IM. Effects that passive cycling exercise have on muscle strength, duration of mechanical ventilation, and length of hospital stay in critically ill patients: a randomized clinical trial. J Bras Pneumol. 2017;43(2):134-9. doi: 10.1590/ s1806-37562016000000170

10. Ely EW, Truman B, Shintani A, Thomason JW, Wheeler AP, Gordon S, et al. Monitoring sedation status over time in ICU patients: reliability and validity of the Richmond AgitationSedation Scale (RASS). JAMA. 2003;289(22):2983-91. doi: 10.1001/jama.289.22.2983

11. Knaus WA, Zimmermann JE, Wagner DP, Draper EA, Lawrence DE. APACHE-acute physiology and chronic health evaluation: a physiologically based classification system. Crit Care Med. 1981;9(8):591-7. doi: 10.1097/00003246-198108000-00008

12. Fivez T, Hendrickx A, Van Herpe T, Vlasselaers D, Desmet L, Van den Berghe $\mathrm{G}$, et al. An analysis of reliability and accuracy of muscle thickness ultrasonography in critically ill children and adults. J Parenter Enteral Nutr. 2016;40(7):944-9. doi: 10.1177/0148607115575033

13. Lindenau JD, Guimarães LSP. Calculando o tamanho de efeito no SPSS. Rev HCPA. 2012;32(3):363-81

14. Lodeserto F, Yende S. Understanding skeletal muscle wasting in critically ill patients. Crit Care. 2014;18(6):617. doi: 10.1186/s13054-014-0617-7

15. Gruther W, Kainberger F, Fialka-Moser V, Paternostro-Sluga T, Quittan M, Spiss C, et al. Effects of neuromuscular electrical stimulation on muscle layer thickness of knee extensor muscles in intensive care unit patients: a pilot study. J Rehabil Med. 2010;42(6):593-7. doi: 10.2340/16501977-0564

16. Renaud G, Llano-Diez M, Ravara B, Gorza L, Feng HZ, Jin JP, et al. Sparing of muscle mass and function by passive loading in an experimental intensive care unit model. J Physiol. 2013;591(5):1385-402. doi: 10.1113/jphysiol.2012.248724

17. Llano-Diez M, Renaud G, Andersson M, Marrero HG, Cacciani $\mathrm{N}$, Engquist $\mathrm{H}$, et al. Mechanisms underlying ICU muscle wasting and effects of passive mechanical loading. Crit Care. 2012;16(5):209. doi: 10.1186/cc11841

18. Pavlov VA, Tracey KJ. The cholinergic anti-inflammatory pathway. Brain Behav Immun. 2005;19(6):493-9. doi: 10.1016/j. bbi.2005.03.015

19. Strasser EM, Stättner S, Karner J, Klimpfinger M, Freynhofer M, Zaller V, et al. Neuromuscular electrical stimulation reduces skeletal muscle protein degradation and stimulates insulinlike growth factors in an age and current dependent manner: a randomized, controlled clinical trial in major abdominal surgical patients. Ann Surg. 2009;249(5):738-43. doi: 10.1097/SLA.0b013e3181a38e71 
20. Maffiuletti NA, Roig M, Karatzanos E, Nanas S. Neuromuscular electrical stimulation for preventing skeletal-muscle weakness and wasting in critically ill patients: a systematic review. BMC Med. 2013;11:137. doi: 10.1186/1741-7015-11-137

21. Burtin C, Clerckx B, Robbeets C, Ferdinande P, Langer $D$, Troosters T, et al. Early exercise in critically ill patients enhances short-term functional recovery. Crit Care Med. 2009;37(9):2499-505. doi: 10.1097/CCM.0b013e3181a38937

22. Puthucheary ZA, Montgomery H, Moxham J, Harridge S, Hart N. Structure to function: muscle failure in critically ill patients. J Physiol. 2010;588(23):4641-8. doi: 10.1113/jphysiol.2010.197632

23. Sarwal A, Parry SM, Berry MJ, Hsu FC, Lewis MT, Justus NW, et al. Interobserver reliability of quantitative muscle sonographic analysis in the critically ill population. J Ultrasound Med. 2015;34(7):1191-200. doi: 10.7863/ultra.34.7.1191

24. Paris M. Mourtzakis M. Assessment of skeletal muscle mass in critically ill patients: considerations for the utility of computed tomography imaging and ultrasonography. Curr Opin Clin Nutr Metab Care. 2016;19(2):125-30. doi: 10.1097/MCO.0000000000000259
25. Segers J, Hermans G, Charususin N, Fivez T, Vanhorebeek I, Van den Berghe $\mathrm{G}$, et al. Assessment of quadriceps muscle mass with ultrasound in critically ill patients: intra- and inter-observer agreement and sensitivity. Intensive Care Med. 2015;41(3):562-3. doi: 10.1007/s00134-015-3668-6

26. Tillquist M, Kutsogiannis DJ, Wischmeyer PE, Kummerlen C, Leung $\mathrm{R}$, Stollery $\mathrm{D}$, et al. Bedside ultrasound is a practical and reliable measurement tool for assessing quadriceps muscle layer thickness. J Parenter Enteral Nutr. 2014;38(7):886-90. doi: 10.1177/0148607113501327

27. Paris MT, Mourtzakis M, Day A, Leung R, Watharkar S, Kozar R, et al. Validation of bedside ultrasound of muscle layer thickness of the quadriceps in the critically ill patient (VALIDUM study). J Parenter Enteral Nutr. 2017;41(2):171-80. doi: 10.1177/0148607116637852

28. Hermans G, Wilmer A, Meersseman W, Milants I, Wouters PJ, Bobbaers $\mathrm{H}$, et al. Impact of intensive insulin therapy on neuromuscular complications and ventilator-dependency in the Medical Intensive Care Unit. Am J Respir Crit Care Med. 2007;175(5):480-9. doi: https://doi.org/10.1164/ rccm.200605-6650C 